\title{
Effect of shear and rotatory inertia on the bending vibration method without weighing specimens
}

\author{
Yoshitaka Kubojima ${ }^{1 *}$, Satomi Sonoda ${ }^{2}$, Hideo Kato ${ }^{1}$ and Masaki Harada ${ }^{1}$
}

\begin{abstract}
This study examines the effect of shear and rotatory inertia on the accuracy of the vibration method with additional mass (VAM). Bending vibration tests were performed for rectangular bars with a width of $30 \mathrm{~mm}$, thicknesses ranging from 5 to $60 \mathrm{~mm}$, and a length of $300 \mathrm{~mm}$, small round bars with diameters in the range of 6-36 mm, and lengths of 150-300 mm, and cross beams for timber guardrails with and without a concentrated mass under a free-free condition. The estimation accuracy of the VAM and the effect of shear and rotatory inertia increased and decreased, respectively, as the length/thickness ratio of the rectangular bar and length/diameter ratio of the round bar increased. The estimation accuracy of the VAM decreased with an increase in the effect of shear and rotatory inertia, and it could be corrected. The range of the effect of shear and rotatory inertia for the sufficient estimation accuracy of the VAM was obtained.
\end{abstract}

Keywords: Bending vibration, Rotatory inertia, Shear, Specimen dimension, Vibration method with additional mass

\section{Introduction}

The vibration test is widely used because it enables Young's modulus to be measured simply, quickly, and non-destructively. Density is a necessary input for calculating the Young's modulus. Weighing specimens can be challenging for certain materials, such as each piled lumber and each cross-beam of timber guardrails. Therefore, a measuring method that does not require specimens to be weighed when measuring Young's modulus would represent a significant practical improvement.

A method for measuring mass, density, and Young's modulus without needing to weigh the specimen was developed based on a frequency equation that incorporates the effect of an additional mass attached to a wooden bar [1-6]. This method is referred to as the vibration method with additional mass (VAM) in this study. This method utilizes decreases in the resonance

\footnotetext{
${ }^{*}$ Correspondence: kubojima@ffpri.affrc.go.jp

${ }^{1}$ Forestry and Forest Products Research Institute, 1 Matsunosato, Tsukuba, Ibaraki 305-8687, Japan

Full list of author information is available at the end of the article
}

frequency by attaching additional mass. The ratio of the resonance frequency with the additional mass to that without it is used for the frequency equation, incorporating the effect of the concentrated mass attached on a specimen and its position. The mass ratio (concentrated mass/specimen) is subsequently calculated from the frequency equation.

To analyze the potential application of the VAM for practical purposes, a series of parameters were investigated, including the suitable mass ratio (additional mass/ specimen) [7], the connection between the additional mass and specimen [7], the crossers' position for the piled lumber [8], the specimen moisture content [9], and the bending vibration generation method [10]. The VAM could perhaps be used to assess the deterioration of the cross beams of timber guardrails [11].

Although the VAM in conjunction with the bending vibration may be effective for piled lumber [12] and the cross beams of timber guardrails [11], the apparent deflection in the bending vibration consists of deflections due to shear and rotatory inertia, as well as pure bending deflection. Hence, this study aims to investigate the effect 
of shear and rotatory inertia on the estimation accuracy of the VAM.

\section{Theory}

\section{Vibration testing method without weighing the specimen}

Here, bending vibrations under free-free conditions are considered. In the case of a thin beam with constant cross section, the effect of deflections due to shear and rotatory inertia involved in the bending vibrational deflection (hereafter, SR effect) is negligible, and the Euler-Bernoulli elementary theory of bending can be applied to the bending vibration.

The resonance frequency, represented by $f_{n 0}$ ( $n$ : resonance mode number, 0 : value without the additional mass), is expressed as follows:

$$
f_{n 0}=\frac{1}{2 \pi}\left(\frac{m_{n 0}}{l}\right)^{2} \sqrt{\frac{E I}{\rho A}},
$$

where $l, E, \rho, I$, and $A$ are the specimen length, Young's modulus, density, the second moment of area, and the cross-sectional area, respectively. $m_{n 0}$ is a constant that depends on the end conditions and is expressed as follows:

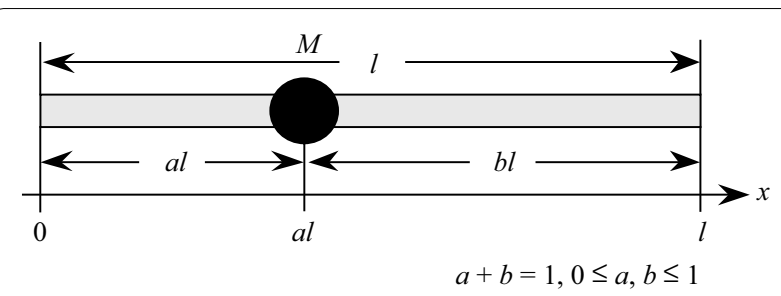

Fig. 1 Beam with additional mass

where $\mu$ is the ratio of the concentrated mass to the mass of the bar and is defined as

$$
\mu=\frac{M}{\rho A l} \text {. }
$$

The measured resonance frequencies $f_{n 0}$ and $f_{n}$ are substituted into Eq. (4) to calculate $m_{n}$, and the calculated $m_{n}$ is substituted into Eq. (5) to calculate $\mu$. The specimen mass and density can be obtained by substituting the calculated $\mu$, the concentrated mass, and the dimensions of a bar into Eq. (6). Young's modulus can be calculated by substituting the estimated density, the resonance frequency without the

$$
m_{10}=4.730, m_{20}=7.853, m_{30}=10.996, m_{n 0}=\frac{1}{2}(2 n+1) \pi(n>3) .
$$

The resonance frequency is decreased experimentally by attaching the additional mass, while the dimensions, density, and Young's modulus are not altered. Hence, it can be said that $m_{n 0}$ changes to $m_{n}$. The resonance frequency after attaching the additional mass is expressed as follows:

$$
f_{n}=\frac{1}{2 \pi}\left(\frac{m_{n}}{l}\right)^{2} \sqrt{\frac{E I}{\rho A}} .
$$

From Eqs. (1) and (3),

$$
m_{n}=\sqrt{\frac{f_{n}}{f_{n 0}}} m_{n 0} .
$$

The frequency equation for the free-free vibration with the concentrated mass $M$ placed at position $x=a l$ ( $x$ : distance along the bar, $0 \leq a \leq 1, a+b=1$ ) on a bar (Fig. 1) is expressed as follows: concentrated mass, and the dimensions of a bar into Eq. (1) [1-6].

The above steps represent the calculation procedure for the VAM. Weighing the specimen is not required for the calculations.

\section{Goens-Hearmon regression method based on the Timoshenko theory of bending (TGH method)}

Young's and shear moduli can be obtained simultaneously using only the bending vibration test without a torsional vibration test by the following the Goens-Hearmon regression method based on the Timoshenko theory of bending (TGH method) [13-15].

The apparent deflection in the bending vibration consists of deflections due to shear and rotatory inertia, as well as pure bending deflection. Timoshenko added the terms deflections of shear and rotatory inertia to the Euler-Bernoulli elementary theory of bending and developed the following differential equation of bending [13]:

$$
\begin{aligned}
\left(\cos m_{n} \cosh m_{n}-1\right)- & \frac{1}{2} \mu m_{n}\left\{\left(\cos a m_{n} \cosh a m_{n}+1\right)\left(\sin b m_{n} \cosh b m_{n}-\cos b m_{n} \sinh b m_{n}\right)\right. \\
& \left.+\left(\cos b m_{n} \cosh b m_{n}+1\right)\left(\sin a m_{n} \cosh a m_{n}-\cos a m_{n} \sinh a m_{n}\right)\right\}=0,
\end{aligned}
$$




$$
\frac{E I}{\rho A} \frac{\partial^{4} y}{\partial x^{4}}+\frac{\partial^{2} y}{\partial t^{2}}-\frac{I}{A}\left(1+\frac{s E}{G}\right) \frac{\partial^{4} y}{\partial x^{2} \partial t^{2}}+\frac{\rho s I}{G A} \frac{\partial^{4} y}{\partial t^{4}}=0,
$$

where $G$ is the shear modulus, $y$ is the lateral deflection, and $t$ is the time.

$$
B_{\mathrm{t}}=\frac{I}{2 l^{2} A}\left(-1+\frac{s E}{G}\right)
$$

Goens approximated Eqs. (9) and (10) using a Taylor series into the following formula [14]:

$$
\frac{m_{n 0}^{4}}{k_{n 0}^{4}}=\frac{E}{E_{\mathrm{a} n}}=T=1+\frac{I}{l^{2} A}\left\{m_{n 0}^{2} F^{2}\left(m_{n 0}\right)+6 m_{n 0} F\left(m_{n 0}\right)\right\}+\frac{I}{l^{2} A} \frac{s E}{G}\left\{m_{n 0}^{2} F^{2}\left(m_{n 0}\right)-2 m_{n 0} F\left(m_{n 0}\right)\right\}-\frac{4 \pi^{2} \rho s I f_{n 0}^{2}}{G A}
$$

where

When Eq. (7) is solved under the free-free condition, the resonance frequency corresponding to the $n$th mode $f_{\mathrm{g} n 0}$ can be written as follows:

$$
F\left(m_{10}\right)=0.9825, F\left(m_{20}\right)=1.0008, F\left(m_{n 0}\right)=1(n>2) .
$$

Approximately,

$$
T=1+\frac{I}{l^{2} A}\left[\left\{m_{n 0}^{2} F^{2}\left(m_{n 0}\right)+6 m_{n 0} F\left(m_{n 0}\right)\right\}+\frac{s E}{G}\left\{m_{n 0}^{2} F^{2}\left(m_{n 0}\right)-2 m_{n 0} F\left(m_{n 0}\right)\right\}\right] .
$$

$$
\begin{aligned}
& f_{\mathrm{g} n 0}=\frac{1}{2 \pi}\left(\frac{k_{n 0}}{l}\right)^{2} \sqrt{\frac{E I}{\rho A}}=\frac{1}{2 \pi}\left(\frac{m_{n 0}}{l}\right)^{2} \sqrt{\frac{E_{\mathrm{a} n} I}{\rho A}},(8) \quad \begin{array}{r}
\text { Hearm } \\
\text { dure after }
\end{array} \\
& Y=E_{\mathrm{a} n}\left[1+\frac{I}{l^{2} A}\left\{m_{n 0}^{2} F^{2}\left(m_{n 0}\right)+6 m_{n 0} F\left(m_{n 0}\right)\right\}-\frac{4 \pi^{2} \rho s I f_{n 0}^{2}}{G A}\right],
\end{aligned}
$$

where $E_{\mathrm{a} n}$ is Young's modulus from the Euler-Bernoulli elementary theory of bending using the resonance frequency of the $n$th mode.

$k_{n 0}$ in Eq. (8) is obtained by transcendental equations represented as follows:

$$
\begin{aligned}
& X=E_{\mathrm{a} n} \frac{I}{l^{2} A}\left\{m_{n 0}^{2} F^{2}\left(m_{n 0}\right)-2 m_{n 0} F\left(m_{n 0}\right)\right\} \\
& \alpha=\frac{s E}{G}
\end{aligned}
$$

$$
\frac{\tan \frac{k_{n 0}}{2} \sqrt{\sqrt{B_{\mathrm{t}}^{2} k_{n 0}^{4}+1}+A_{\mathrm{t}} k_{n 0}^{2}}}{\tanh \frac{k_{n 0}}{2} \sqrt{\sqrt{B_{\mathrm{t}}^{2} k_{n 0}^{4}+1}-A_{\mathrm{t}} k_{n 0}^{2}}}=-\frac{\sqrt{\sqrt{B_{\mathrm{t}}^{2} k_{n 0}^{4}+1}+A_{\mathrm{t}} k_{n 0}^{2}}}{\sqrt{\sqrt{B_{\mathrm{t}}^{2} k_{n 0}^{4}+1}-A_{\mathrm{t}} k_{n 0}^{2}}} \frac{\sqrt{B_{\mathrm{t}}^{2} k_{n 0}^{4}+1}-B_{\mathrm{t}} k_{n 0}^{2}}{\sqrt{B_{\mathrm{t}}^{2} k_{n 0}^{4}+1}+B_{\mathrm{t}} k_{n 0}^{2}}(\text { for symmetric modes}),
$$

and

$$
\beta=E,
$$

$$
\frac{\cot \frac{k_{n 0}}{2} \sqrt{\sqrt{B_{\mathrm{t}}^{2} k_{n 0}^{4}+1}+A_{\mathrm{t}} k_{n 0}^{2}}}{\operatorname{coth} \frac{k_{n 0}}{2} \sqrt{\sqrt{B_{\mathrm{t}}^{2} k_{n 0}^{4}+1}-A_{\mathrm{t}} k_{n 0}^{2}}}=+\frac{\sqrt{\sqrt{B_{\mathrm{t}}^{2} k_{n 0}^{4}+1}+A_{\mathrm{t}} k_{n 0}^{2}}}{\sqrt{\sqrt{B_{\mathrm{t}}^{2} k_{n 0}^{4}+1}-A_{\mathrm{t}} k_{n 0}^{2}}} \frac{\sqrt{B_{\mathrm{t}}^{2} k_{n 0}^{4}+1}-B_{\mathrm{t}} k_{n 0}^{2}}{\sqrt{B_{\mathrm{t}}^{2} k_{n 0}^{4}+1}+B_{\mathrm{t}} k_{n 0}^{2}} \text { (for asymmetric modes), }
$$

where

$$
A_{\mathrm{t}}=\frac{I}{2 l^{2} A}\left(1+\frac{s E}{G}\right),
$$

and and then from Eqs. (16)-(19):

$$
Y=-\alpha X+\beta .
$$

Therefore, the linear regression between $X$ and $Y$ provides the $E$ and $G$ values. This is the Goens-Hearmon regression method based on the Timoshenko theory of 
bending (TGH method). The value of $s$ is $6 / 5$ theoretically [16] and 1.18 experimentally [17] for the rectangular cross section and 10/9 [16] for the circular cross section.

\section{Materials and methods}

\section{Specimens}

Sitka spruce (Picea sitchensis Carr.) rectangular bars with a width of $30 \mathrm{~mm}$ (radial direction, R), thicknesses of $5,10,15,20,25,30,35,40,45,50,55$, and $60 \mathrm{~mm}$ (tangential direction, $\mathrm{T}$ ), and a length of $300 \mathrm{~mm}$ (longitudinal direction, $\mathrm{L}$ ) were used as the specimens. Japanese cedar (Cryptomeria japonica D. Don) small round bars without a pith with varying diameters of $6,9,20,25,30$, and $36 \mathrm{~mm}$ and a length of $300 \mathrm{~mm}$, and those with a diameter of $36 \mathrm{~mm}$ and varying lengths of 150, 200, and $250 \mathrm{~mm}$ were used as the specimens. Three specimens were made for each dimension. Three Japanese cedar cross beams for timber guardrails with a pith having a diameter of $200 \mathrm{~mm}$ and a length of $1980 \mathrm{~mm}$ [11] were also used as the specimens. Considering the variation in the results of the vibration test, the number of specimens was three.

The specimens were conditioned at $20^{\circ} \mathrm{C}$ and $65 \%$ relative humidity. All tests were conducted under the same conditions.

\section{Vibration tests}

Free-free bending vibration tests were conducted to measure the resonance frequencies of the first to fifth resonance modes using the following procedure: the test bar without a concentrated mass was suspended by two threads at the nodal positions of the free-free vibration, corresponding to each resonance mode. The bending

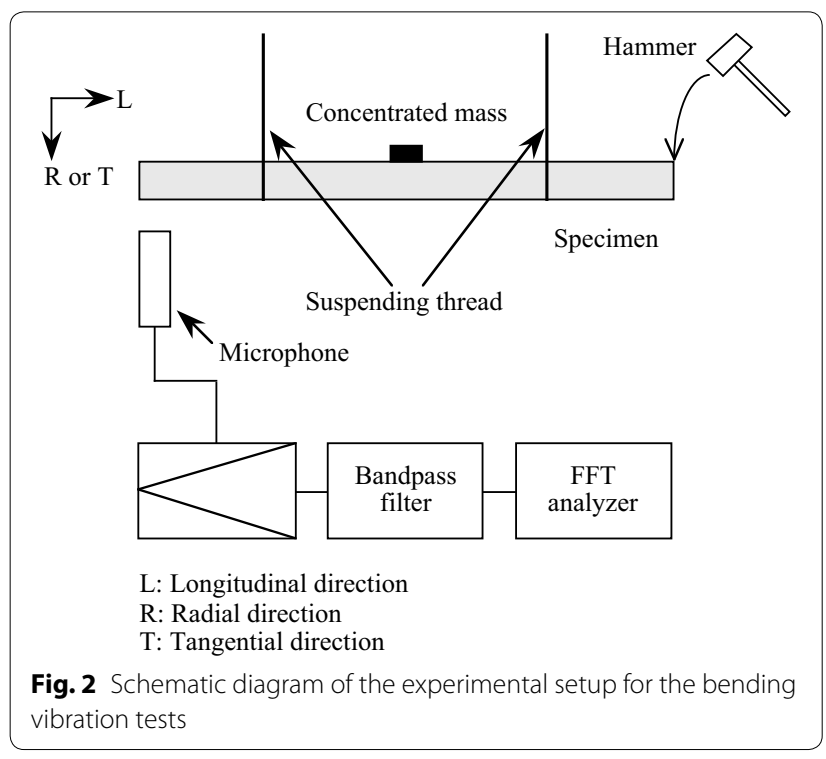

vibration was subsequently generated by tapping the LR-plane of the rectangular bars and the small round bars in the T-direction and it was generated by tapping the LT-plane of the cross beams for timber guardrails in the R-direction, using a wooden hammer. The motion of the specimen was detected with a microphone. The signal was processed through a fast Fourier transform (FFT) digital signal analyzer (Multi-Purpose FFT Analyzer CF-5220, Ono-Sokki, Co., Ltd., Yokohama, Japan) to yield the high-resolution resonance frequencies. A diagram of the experimental setup is provided in Fig. 2.

The free-free bending vibration tests were also performed on the specimens with the concentrated mass using the procedure described above and the resonance frequency of the first mode was measured. Iron plates, thumbtacks, and wood screws were used as the concentrated mass. The position of the concentrated mass was $x=0.5 l$, considering the on-site quality evaluation of cross beams of wooden guardrails. The concentrated masses and specimens are shown in Tables 1 and 2.

\section{Results and discussion}

The mean (standard deviation) density and Young's modulus of the specimens were $449(14) \mathrm{kg} / \mathrm{m}^{3}, 14.23$ (0.45) GPa (sitka spruce rectangular bars; TGH method), and $351(16) \mathrm{kg} / \mathrm{m}^{3}, 10.05$ (1.04) GPa (Japanese cedar small round bars; TGH method), respectively.

The estimation accuracy of the VAM, which is expressed by the mass ratio (estimated specimen mass through the VAM/measured specimen mass), increased as the length/ thickness ratio of the rectangular bar and the length/diameter ratio of the small round bar increased, as shown in Fig. 3. Conversely, the effect of deflections due to shear and

Table 1 Concentrated masses (iron plates) attached to rectangular bars

\begin{tabular}{|c|c|c|c|}
\hline \multicolumn{2}{|c|}{ Concentrated mass } & \multirow{2}{*}{$\begin{array}{l}\text { Specimens } \\
\text { Dimensions }\end{array}$} & \multirow[t]{2}{*}{ Mass ratio $\mu$} \\
\hline Dimensions & Mass & & \\
\hline $2 \times 2 \times 30$ & 1.0 & $30 \times 5 \times 300$ & 0.0453 \\
\hline $4 \times 2 \times 30$ & 2.0 & $30 \times 10 \times 300$ & 0.0474 \\
\hline $7 \times 2 \times 30$ & 3.2 & $30 \times 15 \times 300$ & 0.0504 \\
\hline $9 \times 2 \times 30$ & 4.1 & $30 \times 20 \times 300$ & 0.0492 \\
\hline $11 \times 2 \times 30$ & 5.1 & $30 \times 25 \times 300$ & 0.0524 \\
\hline $13 \times 2 \times 30$ & 6.0 & $30 \times 30 \times 300$ & 0.0518 \\
\hline $14 \times 2 \times 30$ & 7.0 & $30 \times 35 \times 300$ & 0.0512 \\
\hline $17 \times 2 \times 30$ & 7.9 & $30 \times 40 \times 300$ & 0.0506 \\
\hline $20 \times 2 \times 30$ & 9.4 & $30 \times 45 \times 300$ & 0.0515 \\
\hline $21 \times 2 \times 30$ & 10.3 & $30 \times 50 \times 300$ & 0.0510 \\
\hline $24 \times 2 \times 30$ & 11.3 & $30 \times 55 \times 300$ & 0.0506 \\
\hline $26 \times 2 \times 30$ & 12.2 & $30 \times 60 \times 300$ & 0.0507 \\
\hline
\end{tabular}

Unit of dimensions: $\mathrm{mm}$, unit of mass: $\mathrm{g}$ 
Table 2 Concentrated masses (thumbtacks, wood screws) attached to small round bars

\begin{tabular}{|c|c|c|c|c|c|c|}
\hline \multicolumn{5}{|c|}{ Concentrated mass } & \multirow{2}{*}{$\begin{array}{l}\text { Specimens } \\
\text { Diameter, length }\end{array}$} & \multirow[t]{2}{*}{ Mass ratio $\mu$} \\
\hline Type & Head diameter & Nominal diameter & Length & Mass & & \\
\hline Thumbtack & 11 & 1 & 8 & 0.6 & $\begin{array}{l}6,300 \\
9,300\end{array}$ & $\begin{array}{l}0.214 \\
0.0905\end{array}$ \\
\hline Wood screw & 6 & 4 & 32 & 2.1 & $\begin{array}{l}20,300 \\
25,300 \\
36,150\end{array}$ & $\begin{array}{l}0.0615 \\
0.0437 \\
0.0393\end{array}$ \\
\hline Wood screw & 9 & 5 & 37 & 3.6 & $\begin{array}{l}30,300 \\
36,200 \\
36,250\end{array}$ & $\begin{array}{l}0.0503 \\
0.0485 \\
0.0395\end{array}$ \\
\hline Wood screw & 8 & 4 & 65 & 3.9 & 36,300 & 0.0392 \\
\hline
\end{tabular}

Units of diameter and length: $\mathrm{mm}$, unit of mass: $\mathrm{g}$
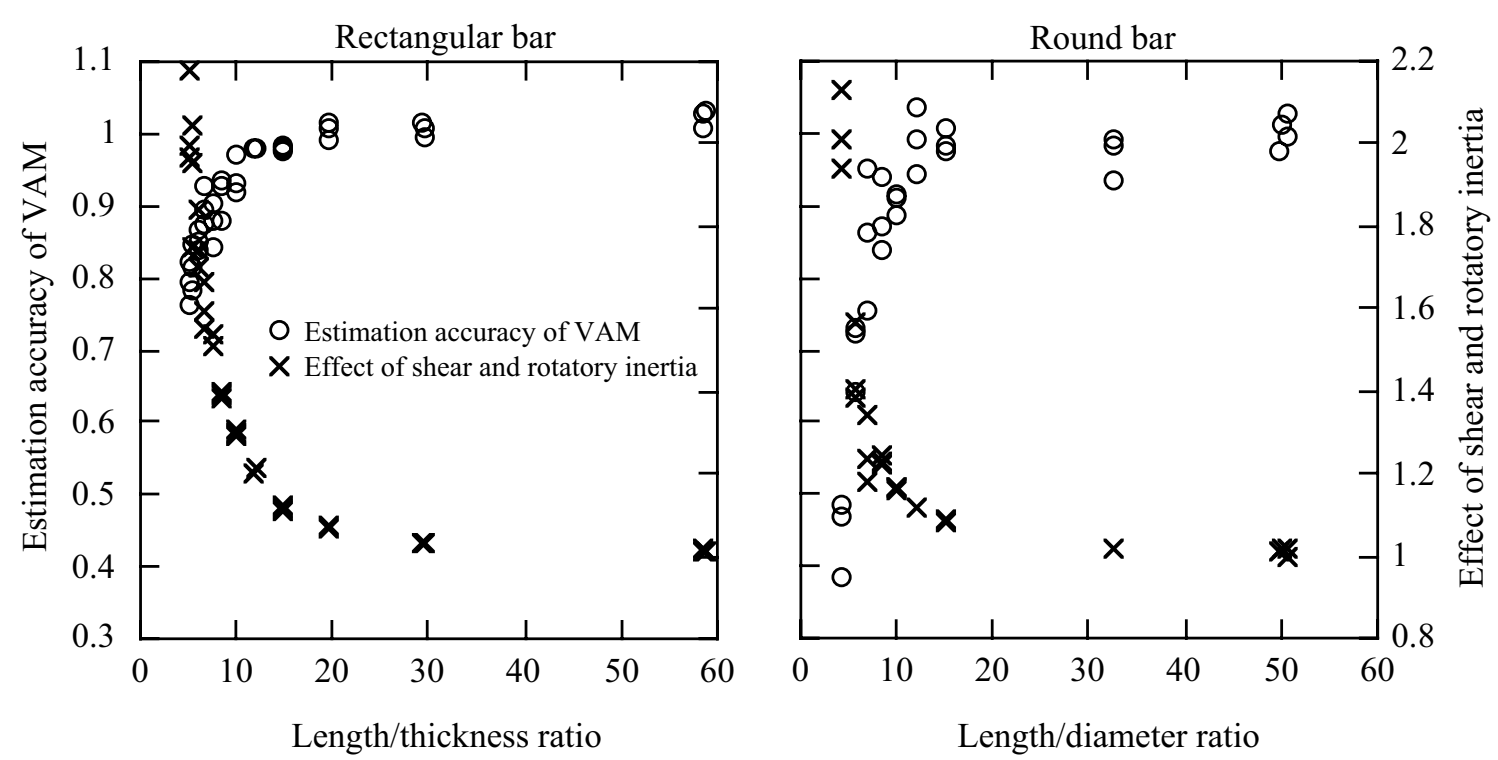

Fig. 3 Estimation accuracy of the VAM and the effect of deflections due to shear and rotatory inertia involved in the bending vibrational deflection (SR effect) at various length/thickness and length/diameter ratios

rotatory inertia involved in the bending vibration deflection (SR effect) shown by $T\left(E_{\mathrm{a} n}\right.$ is calculated from the first resonance mode in this study) in Eq. (13) decreased as the length/thickness and length/diameter ratios increased, as shown in Fig. 3. Thus, the estimation accuracy of the VAM is discussed from the aspect of $T$.

When the SR effect is taken into consideration, the resonance frequency without the concentrated mass is expressed by Eq. (8), and the resonance frequency with the concentrated mass is expressed as follows:

$$
f_{\mathrm{g} n}=\frac{1}{2 \pi}\left(\frac{k_{n}}{l}\right)^{2} \sqrt{\frac{E I}{\rho A}} .
$$

From Eqs. (8) and (21),

$$
k_{n}=\sqrt{\frac{f_{\mathrm{g} n}}{f_{\mathrm{g} n 0}}} k_{n 0}=\sqrt{\frac{f_{\mathrm{g} n}}{f_{\mathrm{g} n 0}}} \frac{m_{n 0}}{\sqrt[4]{T}} .
$$

When the SR effect can be ignored, the $m_{n}$ decreases monotonically with $\mu$ for the free-free vibration [5]. Hence, the specimen mass estimated by the VAM is low for a low $m_{n}$ from Eq. (6). Analogizing this, when $k_{n}$ decreases, $\mu$ increases, and the specimen mass decreases, which causes the estimation accuracy of the VAM to decrease. Equation (22) shows that $k_{n}$ decreases with an increasing $T$. Therefore, when $T$ increases with the decreases in the length/thickness and length/diameter ratios, $k_{n}$ decreases, causing the estimation accuracy of the VAM to decrease. 
As the SR effect is not taken into consideration in Eq. (5), $k_{n}$ cannot be directly substituted for Eq. (5). In other words, $f_{n} / f_{n 0}$ needed to be used instead of $f_{\mathrm{g} n} / f_{\mathrm{g} n 0}$ for Eq. (4). With the assumption that the SR effect changes $f_{n} / f_{n 0}$ to $f_{\text {gn }} / f_{\text {gn } 0}$, the relationship between $\left(f_{\mathrm{g} n} / f_{\mathrm{g} n 0}\right) /\left(f_{n} / f_{n 0}\right)$ and $T$ was investigated. For this purpose, $\left(f_{\mathrm{g} n} / f_{\mathrm{g} n 0}\right) /\left(f_{n} / f_{n 0}\right)$ was plotted against $T . m_{n}$ was calculated by substituting $\mu$, which is the ratio of the measured concentrated mass to the specimen mass, for Eq. (5), and $f_{n} / f_{n 0}$ was then obtained from Eqs. (2) and (4). Mathematica (version 10.4 J, Wolfram Research Co., Ltd.) was used for solving Eq. (5). $\left(f_{\mathrm{g} n} / f_{\mathrm{g} n 0}\right) /\left(f_{n} / f_{n 0}\right)$ $\left(f_{\mathrm{g} n}\right.$ and $f_{\mathrm{g} n 0}$ are measured values) linearly decreased with an increasing $T$, as shown in Fig. 4 and the regression equations of Eqs. (23) and (24) were obtained: $m_{n}$ was subsequently obtained. The specimen mass was estimated by substituting the obtained $m_{n}$ for Eq. (5). The results showed that the estimation accuracy of the VAM was improved, as shown in Fig. 5. The estimation accuracy of the VAM could not be improved in several cases. In such cases, $\left(f_{\mathrm{g} n} / f_{\mathrm{g} n 0}\right) /\left(f_{n} / f_{n 0}\right)$ was out of lines that are shown by Fig. 4 and Eqs. (23) and (24).

As mentioned above, $T=E / E_{\mathrm{a} n}$ is necessary to estimate $f_{n} / f_{n 0}$ from $f_{\mathrm{g} n} / f_{\mathrm{g} n 0}$. In other words, the bending Young's modulus based on the Euler-Bernoulli elementary theory, which can be calculated by measuring one resonance mode, and the "true" Young's modulus without the SR effect are required. The true Young's modulus can be measured through the TGH method or the longitudinal vibration test. As the resonance frequen-

$$
\begin{aligned}
& \frac{f_{\mathrm{g} n} / f_{\mathrm{g} n 0}}{f_{n} / f_{n 0}}=-0.0084 T+1.01\left(r=0.972^{* *}, n=1\right) \text { for the rectangular bar, } \\
& \frac{f_{\mathrm{g} n} / f_{\mathrm{g} n 0}}{f_{n} / f_{n 0}}=-0.030 T+1.03\left(r=0.956^{* *}, n=1\right) \text { for the small round bar. }
\end{aligned}
$$

The estimation accuracy of the VAM is corrected with the following method. $f_{n} / f_{n 0}$ was estimated by substituting the measured $T=E / E_{\text {an }}$ (TGH method/Euler-Bernoulli elementary theory) and measured $f_{\mathrm{g} n} / f_{\mathrm{g} n 0}$ for Eqs. (23) and (24). The estimated $f_{n} / f_{n 0}$ was used for Eq. (4), and cies of plural vibration modes are required, obtaining $T=E / E_{\mathrm{a} n}$ on-site, where simplicity and speed are needed, is challenging.

Since the estimation accuracy of the VAM was high when $T$ was small, as shown in Fig. 3, $T$ that provides a sufficiently high estimation accuracy of VAM is discussed

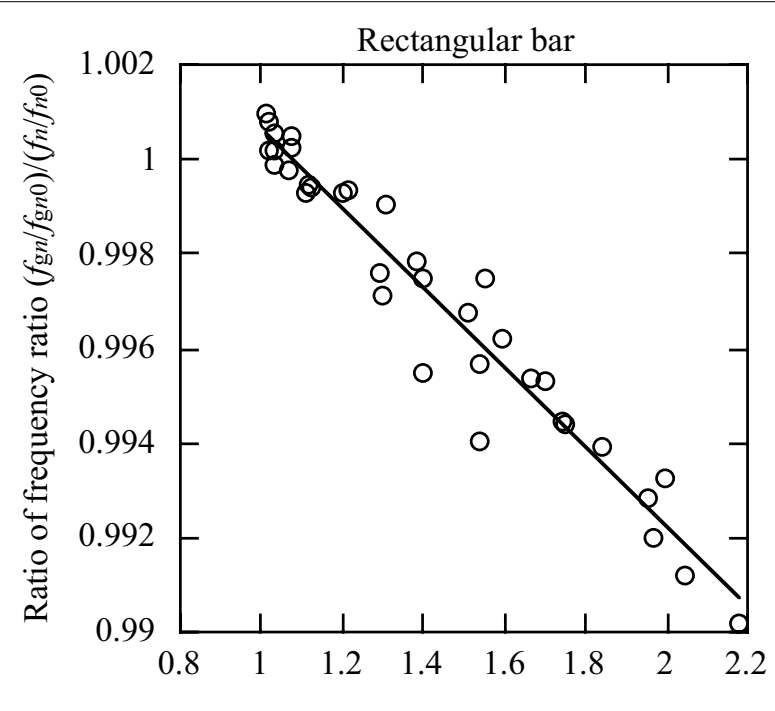

Effect of shear and rotatory inertia

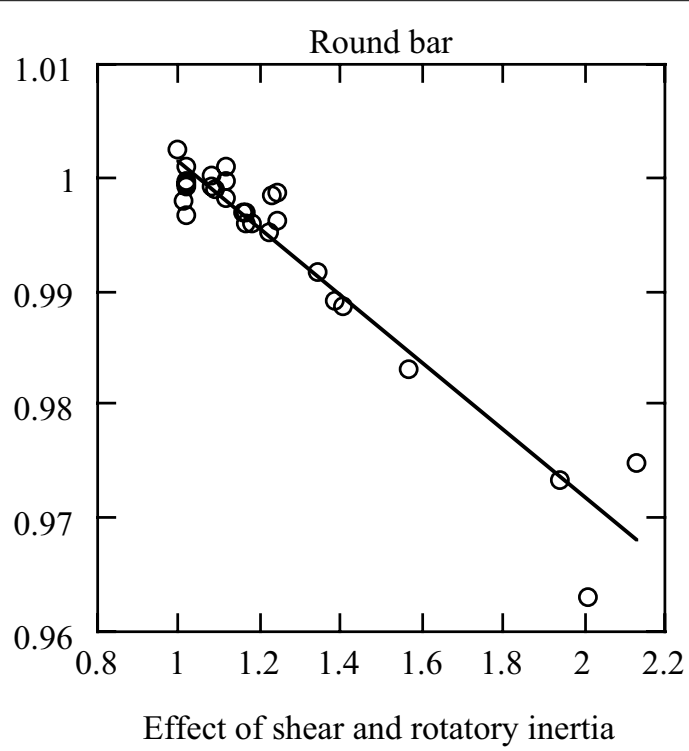

Effect of shear and rotatory inertia

Fig. 4 Relationship between the ratio of frequency ratio $\left(f_{\mathrm{gn}} / f_{\mathrm{gno}}\right) /\left(f_{n} / f_{n 0}\right)$ and the effect of deflections due to shear and rotatory inertia involved in the bending vibrational deflection $T$ 

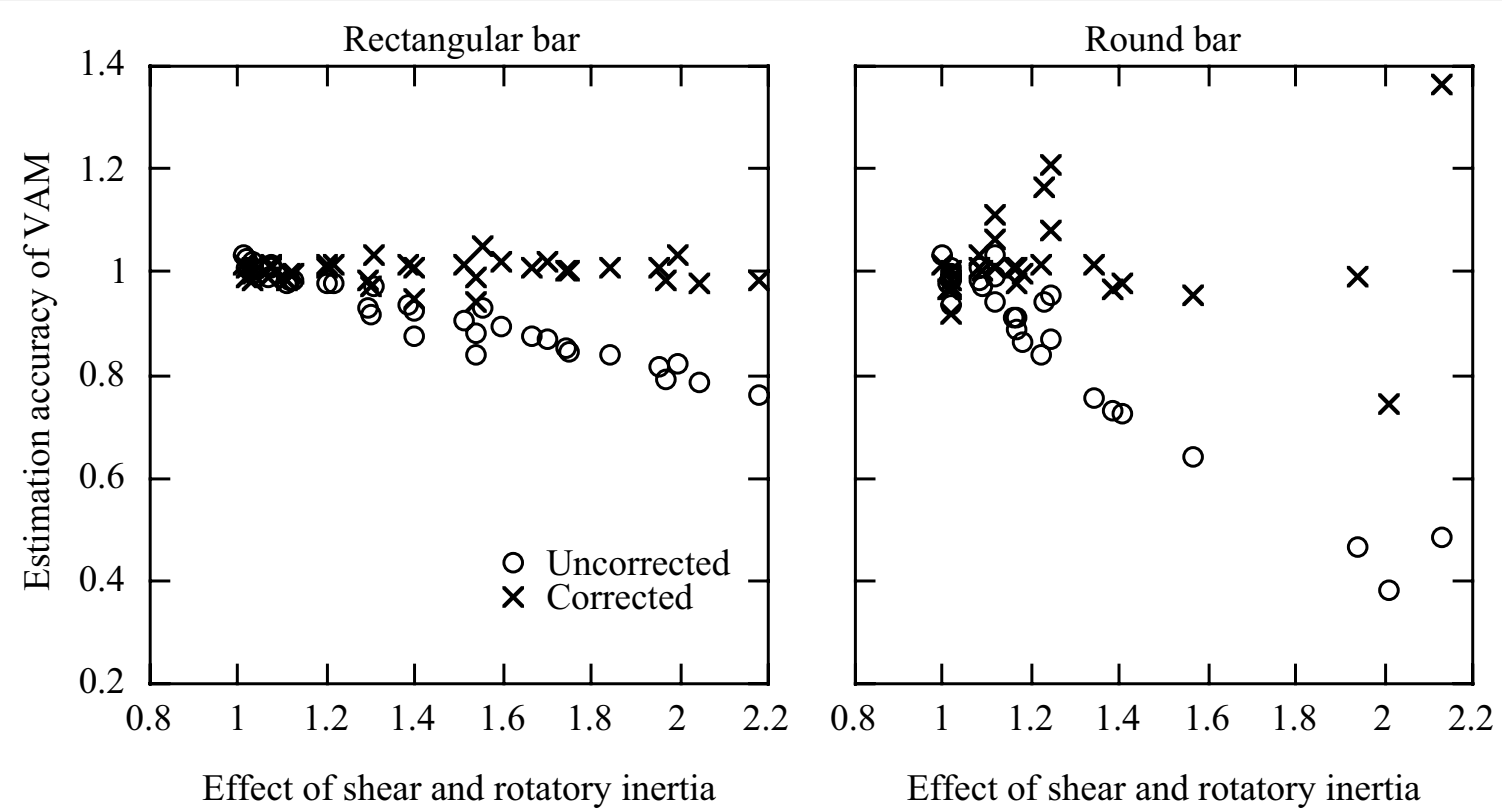

Fig. 5 Relationship between the estimation accuracy of the VAM and the effect of deflections due to shear and rotatory inertia involved in the bending vibrational deflection $T$

here. Based on the coefficient of regression of the TGH method in our previous study, the SR effect was experimentally low in the range of $T \leq 1.2$ [18]. In the range of $T \leq 1.2$, the estimation accuracy of the VAM for 11 out of 11 specimens $(100 \%)$ for rectangular bars and that for 15 out of 17 specimens $(88 \%)$ for small round bars were from 0.9 to 1.1. Hence, it can be said that the estimation accuracy of the VAM is sufficiently high in the range of $T \leq 1.2$. The estimation accuracies of the VAM for cross beams of wooden guardrails were $0.89,0.91$, and 1.06 [11] for $T=1.13,1.11$, and 1.17, respectively. The dimensions of a specimen that give $T \leq 1.2$ can be calculated by substituting $T=1.2$, Young's modulus and shear modulus published in the literature for Eq. (15).

\section{Conclusions}

Bending vibrations were undertaken for rectangular bars and round bars with various dimensions with and without the concentrated mass, and the following results were obtained:

1. The estimation accuracy of the VAM and the SR effect increased and decreased, respectively, as the length/thickness ratio of the rectangular bar and the length/diameter ratio of the round bar increased.

2. The estimation accuracy of the VAM decreased as the SR effect increased.

3. The SR effect on the estimation accuracy of the VAM could be corrected.
4. The range of $T$ for obtaining a sufficiently high accuracy of VAM was $T \leq 1.2$.

\section{Abbreviations}

VAM: Vibration method with additional mass; SR: Shear and rotatory inertia; TGH: Timoshenko-Goens-Hearmon; L: Longitudinal direction; R: Radial direction; T:Tangential direction; FFT: Fast Fourier transform.

\section{Acknowledgements}

This study was supported by Research Grant \#201805 of the Forestry and Forest Products Research Institute.

\section{Authors' contributions}

All authors designed the experiments. YK performed the experiments, analyzed the data, and was a major contributor in writing the manuscript. All authors contributed to interpretation and discussed results. All authors read and approved the final manuscript.

\section{Funding}

This study was supported by Research Grant \#201805 of the Forestry and Forest Products Research Institute.

\section{Availability of data and materials}

All data generated or analyzed during this study are included in this published article.

\section{Competing interests}

The authors declare that they have no competing interests.

\section{Author details}

${ }^{1}$ Forestry and Forest Products Research Institute, 1 Matsunosato, Tsukuba, Ibaraki 305-8687, Japan. ${ }^{2}$ Toyama Prefectural Agricultural, Forestry \& Fisheries Research Center, 4940 Kurokawa Shin, Imizu, Toyama 939-0311, Japan. 
Received: 29 May 2020 Accepted: 17 July 2020

Published online: 25 July 2020

\section{References}

1. Skrinar M (2002) On elastic beams parameter identification using eigenfrequencies changes and the method of added mass. Comput Mater Sci 25:207-217

2. Türker T, Bayraktar A (2008) Structural parameter identification of fixed end beams by inverse method using measured natural frequencies. Shock Vib 15:505-515

3. Kubojima Y, Sonoda S (2015) Measuring Young's modulus of a wooden bar using longitudinal vibration without measuring its weight. Eur J Wood Wood Prod 73:399-401

4. Matsubara M, Aono A, Kawamura S (2015) Experimental identification of structural properties of elastic beam with homogeneous and uniform cross section. Trans JSME. https://doi.org/10.1299/transjsme.15-00279

5. Kubojima Y, Kato H, Tonosaki M, Sonoda S (2016) Measuring Young's modulus of a wooden bar using flexural vibration without measuring its weight. BioResources 11:800-810

6. Matsubara M, Aono A, Ise T, Kawamura S (2016) Study on identification method of line density of the elastic beam under unknown boundary conditions. Trans JSME. https://doi.org/10.1299/transjsme.15-00669

7. Sonoda S, Kubojima Y, Kato H (2016) Practical techniques for the vibration method with additional mass. Part 2: experimental study on the additional mass in longitudinal vibration test for timber measurement. In: CD-ROM proceedings of the world conference on timber engineering (WCTE 2016), Vienna, Austria, August 22-25, 2016

8. Kubojima Y, Sonoda S, Kato H (2017) Practical techniques for the vibration method with additional mass: effect of crossers' position in longitudinal vibration. J Wood Sci 63:147-153
9. Kubojima Y, Sonoda S, Kato H (2017) Practical techniques for the vibration method with additional mass: effect of specimen moisture content. J Wood Sci 63:568-574

10. Kubojima Y, Sonoda S, Kato H (2018) Practical techniques for the vibration method with additional mass: bending vibration generated by tapping cross section. J Wood Sci 64:16-22

11. Kubojima Y, Sonoda S, Kato H (2018) Application of the vibration method with additional mass to timber guardrail beams. J Wood Sci 64:767-775

12. Kubojima Y, Sonoda S, Kato H (2020) Mass of piled lumber estimated through vibration test. J Wood Sci 66:32. https://doi.org/10.1186/s1008 6-020-01880-5

13. Timoshenko SP (1921) On the correction for shear of the differential equation for transverse vibrations of prismatic bars. Philos Mag 6 Ser 41(245):744-746

14. Goens E (1931) Über die Bestimmung des Elastizitätsmodulus von Stäben mit Hilfe von Biegungsschwingungen. Ann Phys 403(6):649-678

15. Hearmon RFS (1958) The influence of shear and rotatory inertia on the free flexural vibration of wooden beams. Br J Appl Phys 9(10):381-388

16. Timoshenko SP, Gere JM (1972) Mechanics of materials. D. Van Nostrand Company, New York

17. Nakao T, Okano T, Asano I (1984) Measurement of anisotropic shear modulus by the tortional-vibration method for free-free wooden beams. Mokuzai Gakkaishi 30:877-885

18. Kubojima Y, Yoshihara H, Ohta M, Okano T (1996) Examination of the method of measuring the shear modulus of wood based on the Timoshenko theory of bending. Mokuzai Gakkaishi 42:1170-1176

\section{Publisher's Note}

Springer Nature remains neutral with regard to jurisdictional claims in published maps and institutional affiliations.

\section{Submit your manuscript to a SpringerOpen ${ }^{\odot}$ journal and benefit from:}

- Convenient online submission

- Rigorous peer review

- Open access: articles freely available online

- High visibility within the field

- Retaining the copyright to your article

Submit your next manuscript at $\boldsymbol{\nabla}$ springeropen.com 